\title{
65822
}

\section{HELIUM ABUNDANCE VARIATIONS}

\section{K. W. OGILVIE}

\section{JANUARY 1972}

\section{GODDARD SPACE FLIGHT CENTER}

GREENBELT, MARYLAND

(NASA-TH-X-65822) HELIUH ABUNDANCE VARIATIONS $18 \mathrm{p}$

K. H. Ogilvie (NASA)

Jan.

CSCL $03 \mathrm{~B}$
N $72-17866$

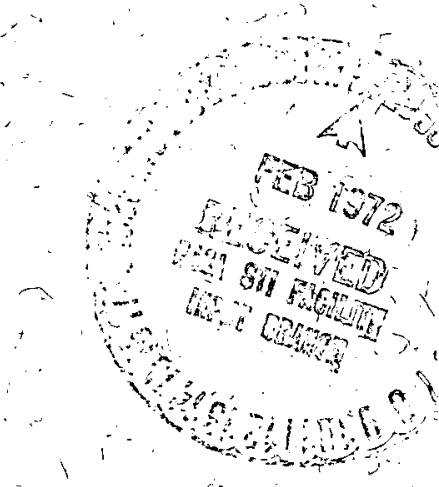


HELIUM ABUNDANCE VARIATIONS

by

K. W. Ogilvie

Laboratory for Extraterrestrial Physics

NASA-Goddard Space Flight Center

Greenbelt, Maryland

January 1972 


\section{Abstract}

Using observations of the solar wind by Explorers 34 and 43, we confirm the relationship between the relative abundance of helium $n_{\alpha} / n_{p}$ and the bulk speed $U$ of the wind,recently described by Hirschberg, Asbridge and Robbins. Use of this relationship $n_{\alpha} / n_{p}=a(t)+b U$ allows the dependence of the relative abundance of helium upon solar activity to be separated from its bulk speed dependence. The proportion of helium in the solar wind at a given bulk speed is found to increase with increasing sunspot number, averaged over the period of observation. We show that the relationship between average relative abundance and temperature, deduced using the bulk speed - temperature relation, is consistent with the data. The relative abundance of helium is not found to increase with increasing solar wind flux. This confirms the result of Hirschberg et. al., and contradicts the theoretical prediction of Geiss. 
Introduction

In this note we discuss and present observations of helium ions in the solar wind made by experiments aboard the satellites Explorer 34 and Explorer 43. Besides presenting new results, we sha11 compare these results with those recently published by Hirschberg et. al。, (1972), obtained from the analysis of data from Vela $3 \mathrm{~A}$ and $3 \mathrm{~B}$.

The relative abundance of helium (the ratio of number densities $\mathrm{n}_{\alpha} / \mathrm{n}_{\mathrm{p}}$ ) in the solar wind is a highly variable quantity, and this variation has been discussed in recent review articles, for example Hundhausen, 1970. It is usual to distinguish between the anomalously high relative abundance of helium sometimes observed in the gas behind driven shock waves (for references to this phenomenon see Hirschberg et. a1., 1970), and the more usual day-to-day variation observed over long periods. The shock-associated increases have been interpreted as observations of helium-enriched plasma from the chromosphere or lower corona (Hirschberg et. al., 1970; Hirschberg, 1971)。 It has been suggested, (Robbins et.al., 1970), that the day-to-day variation is the result of changing conditions in the solar corona and also that the value of the hydrogen to helium ratio, averaged over appropriate times, varies with solar activity.

In a recent note Hirschberg et. al. (1972) have shown that the average relative abundance of helium in the solar wind as measured on Vela 3A and 3B between July 1965 and July 1967 is proportional to the bulk speed of the solar wind, U. Our observations of day-to-day variations confirm this finding. By using data from three periods of the solar cycle, and referring the relative abundance of helium to a fixed bulk speed, we present evidence that the relative abundance is a function of solar activity。 
We also confirm the finding by Hirschberg et. al。, 1972, that the relative abundance of helium does not vary with the solar wind flux in the manner predicted by Geiss et. al., (1970)。Combining the $\left(\mathrm{n}_{\alpha} / \mathrm{n}_{\mathrm{p}}, \mathrm{U}\right)$ relation with the $\left(\mathrm{T}_{\mathrm{p}}^{1 / 2}, \mathrm{U}\right)$ relation of Burlaga and Ogilvie (1970a) we obtain an $\left(\frac{n_{\alpha}}{n_{p}}, T_{p}\right)$ relation which represents the data, and discuss this in connection with the work of Weber (1971). 


\section{Observations}

The observations to be discussed consist of two sets. The first was obtained from a detector on Explorer 43. This instrument is a cylindrical electrostatic analyser, recording 20-point energy-per-charge spectra, and operated between 18 March and 10 April 1971. A spectrum was obtained every four minutes, and values of proton bulk speed, U, density, $n_{p}$, temperature, $T_{p}$, and helium to hydrogen ratio $n_{\alpha} / n_{p}$ were deduced by fitting the observations to the sum of two convected Maxwellian velocity distribution functions. Equal bulk speeds were assumed for each species, (Ogilvie and Zwally, 1971), and the helium temperature was assumed to be four times that of the hydrogen (Robbins et. al., 1970). This assumption is true for a sufficiently large proportion of the time not to be restrictive on the results discussed here. This set consists of 5313 observations.

The second set consists of 637 30-minute averages derived from the 3-minute observations made by Explorer 34. The instrument and method of data reduction have been described before (Ogilvie, Burlaga, and Richardson, 1967). The 30-minute averages are used because the averaging process improves the signal to noise ratio, and thus gives more consistent values of helium density $\mathrm{n}_{\alpha}$. 


\section{$\underline{\text { Results }}$}

Variations of $n / \frac{n}{p}$ with bulk speed and sunspot number.

Figure 1 shows plots of $n_{\alpha} / n_{p}$ vs $U$. These relative abundances are averages over the appropriate observing time for $25 \mathrm{~km} \mathrm{sec}^{-1}$ intervals of $U$. The lower line represents the equation of Hirschberg et. al. (1972) .

$$
\mathrm{n}_{\alpha} / \mathrm{n}_{\mathrm{p}} \times 10^{2}=1.02\left(\mathrm{U} \times 10^{-2}\right)-0.39, \mathrm{U} \text { in } \mathrm{km} \mathrm{sec}{ }^{-1}
$$

The points (crosses) about this line show the observations of the Vela plasma instruments from which it is derived. The middle line represents the equation

$$
\mathrm{n}_{\alpha} / \mathrm{n}_{\mathrm{p}} \times 10^{2}=0.97\left(\mathrm{U} \times 10^{-2}\right)+1.96
$$

derived from a least-squares fit to the circled points from the Explorer 43 data. The standard deviations of the means of the observations are indicated. Errors are not quoted for the coefficients in (2), since the observations are not normally distributed about their mean. The standard deviations are shown to indicate a measure of the scatter of the points. The close agreement between the coefficients of $U$ in these two relations indicates that both sets of data, obtained from two different instruments at different times, are consistent with an equation

$$
\mathrm{n}_{\alpha} / \mathrm{n}_{\mathrm{p}} \times 10^{2}=1.0\left(\mathrm{U} \times 10^{-2}\right)+\mathrm{B} \text {, }
$$

where $B$ is a parameter, which depends upon the time when the observations were made.

The uppermost line represents a fit to the points (triangle) derived from Explorer 34 half-hourly average observations. A least-squares fit gives a slope of 0.9 and an intercept of 3.6 in the units of equation 2 . The line plotted in the figure is not the least-square line, but is the straight line with slope $=1$ which passes between the points with the smallest total deviation. This is done because of the scatter in these points, to 
derive the best value of $B$, which is found to be $B=2.9$. The vertical line at $U=320 \mathrm{~km} \mathrm{sec}{ }^{-1}$, the bulk speed considered by Hundhausen, 1970, to be typical of the quiet solar wind, cuts these 1 ines at values of $\mathrm{n}_{\alpha} / \mathrm{n}_{\mathrm{p}}$ of $0.029,0.05$ and 0.061 respective1y.

We can see from Figure 1 , that the variation of the average values of $\mathrm{n}_{\alpha} / \mathrm{n}_{\mathrm{p}}$ due to the normal bulk speed variations of the solar wind are at least as large as those due to changes in solar activity, and the study of changes due to solar activity is made easier by referring $n_{\alpha} / n_{p}$ to a fixed bulk speed. Robbins et. al. (1970), have previously demonstrated the probable dependence of $\mathrm{n}_{\alpha} / \mathrm{n}_{\mathrm{p}}$ on solar activity, but large fluctuations due presumably to bulk speed changes, obscured it to a considerable extent.

In Figure 2 we plot the three values of the parameter $B$ in equation (3) against the average value of $S$ which characterized the interval of observation. The "error" bars shown are obtained by assuming the variability in the data to be "error", and to be normally distributed. They do not represent errors but rather a measure of the range of the variability of the constant $B$ from day to day. The long period of time represented by the Vela observation, July 1965 to July 1967, occurred during the rising protion of the present solar cycle, and took place during a change in sunspot number from 10 to 90 . The other two data sets were taken dring relatively small ranges in sunspot number. There is clearly an increase in the average $\frac{n_{\alpha}}{n_{p}}$, measured at a fixed bulk speed, with increase of sunspot number.

The three points in Figure 2 are not statistically inconsistent with the straight line shown, which leads to the relation

$$
\frac{n_{\alpha}}{n_{p}} \times 10^{2}=1 \times 10^{-2} U+0.078 \mathrm{~s}-3.9
$$


Note this predicts that for $S=10$, a typical value at solar minimum, $\frac{n_{\alpha}}{n_{p}}=0$ for $U=304 \mathrm{~km} \mathrm{sec}^{-1}$. Although a better fit could be made to these points using a polynomial, the accuracy of the present data does warrant it, and we use the linear fit as an example showing that with more accurately intercalibrated data it would be possible to obtain a relation between $n_{\alpha} / n_{p}, U$ and a parameter describing solar activity.

In considering the above interpretation of Figure 1, we must consider possible systematic errors. The density calibration of plasma instruments is notoriously difficult, (Ness et. al。, 1971), but the ratio $n_{\alpha} / n_{p}$ depends primarily upon $\mathrm{e}_{\mathrm{p}} / \mathrm{e}_{\alpha}$, the ratio of the detection efficiencies for hydrogen and helium ions, which quantities in turn depend upon secondary emission coefficients, preamplifier sensitivities, etc. Thus quantitative details of the picture presented above may be in error, but these errors are estimated to be small enough that qualitatively, the result remains correct. In particular, the variation of $B$ with $S$ is monotonic.

Variation of $\mathrm{n} O / \mathrm{n}$, with flux.

In Figure 3, we present this variation for the three sets of data, each indicated in the same way as before. Any trend existing is downward in all three cases, so we must agree with Hirschberg et, al that either (1), minimum solar wind flux required to ensure the acceleration of helium in the solar corona is too low to result in an observable positive variation of $n_{\alpha} / n_{p}$ with flux, or (2) that the importance of wave particle interactions in the acceleration of helium in the solar wind is enough to modify the treatment of Geiss et. al. The Explorer 43 data is consistent with no variation of $n_{\alpha} / n_{p}$ with flux, but the Explorer 34 data shows a definite fall of $n_{\alpha} / n_{p}$ with increasing flux. High fluxes 
are associated with low speeds (Burlaga and Ogilvie, (1970b), in this data sample, so the effect is internally consistent, and the slope of the $n_{\alpha} / n_{p}-f 1 u x$ dependence may be a function of solar activity. Further observations, particularly near solar minimum, are required.

The variation of $\mathrm{n}_{\alpha} / \mathrm{n}$ with $\mathrm{T}_{\mathrm{p}}$.

By combining the $\left(\mathrm{n}_{\alpha} / \mathrm{n}_{\mathrm{p}}, \mathrm{U}\right)$ relation (3) with the (U, $\left.\mathrm{T}_{\mathrm{p}}\right)$ relation of Burlaga and Ogilvie (1970a), we can eliminate $U$ and predict an $\left(\mathrm{n}_{\alpha} / \mathrm{n}_{\mathrm{p}}, \mathrm{T}_{\mathrm{p}}\right)$ relation, but to do this we must assume a form for $B(S)$. For example if we use the linear relation (4) we can "correct" the Explorer 34 and 43 values to $S=50$ and compare them with the Vela results. Figure 4 shows all three sets of observations, together with the dotted curve corresponding to the use of (4) in the (U, $\left.T_{p}\right)$ relation. The Explorer 34 and Vela results agree quite well both with each other and with the predicted 1ine, while those from Explorer 43 are systematically above it for temperatures above $3 \times 10^{4} o_{K}$. Such temperatures correspond to bulk speeds below $300 \mathrm{~km} \mathrm{sec}^{-1}$, where departures from these relations might be expected to occur, and are difficult to measure due to the finite resolution of the instrument.

Figure 2 shows that the equation (4) does not accurately represent the variation of $B(S)$ with $S$. Presumably a more adequate relationship between the quantity $B$ and solar activity would allow all the results to be represented by a single curve of the form

$$
\frac{\mathrm{n}_{\alpha}}{\mathrm{n}_{\mathrm{p}}} \times 10^{2}=0.28\left(\mathrm{~T}_{\mathrm{p}}^{1 / 2}\right)+\mathrm{f},
$$

where $f$ is a function of $S$. At the present time the data does not warrant more exact empirical fitting. There is also some question as to which solar parameter best represents the variation of solar activity for this purpose. For example, the intensity Ig of the $5303 \AA$ coronal spectral 
line has been used to indicate coronal temperature for the purposes of correlation with cosmic ray studies, Wang (1970). Preliminary comparisons indicate that while S ranges from 50 to 93 for the Vela and Explorer 34 observation periods, Ig ranges from $\sim 45$ units to $\sim 75$ units for the solar 1atitude band \pm 30 degrees.

There remains the possibility that there might exist systematic differences between the values of $n_{\alpha} / n_{p}$ obtained by the various experiments. This might be due to systematic differences in ion detection efficiency measurements by either experimenters. Examination of this question for Explorers 34 and 43 shows that errors of this sort are not large enough to affect the present work. 


\section{Discussion}

The $\left(n_{\alpha} / n_{p}, U\right)$ relationship, together with that between bulk speed and temperature, can illustrate many features of the average behavior of the solar wind. Figure 1, for example, shows that sustained values of $\mathrm{n}_{\alpha} / \mathrm{n}_{\mathrm{p}}$ much above 0.1 are inconsistent, even for periods of high solar activity, with the known range of wind speeds. It also appears that close to solar minimum, when values of $S$ of about 10 are normal, the proportion of helium should be very low $(<0.01)$ when the wind speed is $\simeq 320$ $\mathrm{km} \sec ^{-1}$. If equation 4 was taken 1 iterally, $\mathrm{n}_{\alpha} / \mathrm{n}_{\mathrm{p}}$ might go to zero for low
bulk speeds. Attempts to correlate. the average or most probable values of $\mathrm{n}_{\alpha} / \mathrm{n}_{\mathrm{p}}$, observed over a time of several solar rotations, with solar activity have not been satisfactory. This is because the variation of $\mathrm{n}_{\alpha} / \mathrm{n}_{\mathrm{p}}$ with solar activity has been obscured by the equally large variation with wind speed, and because the precise average or most probable value obtained depended upon the proportion of high and low speed observations in the samples upon which they were based.

Sustained departures from such simple relations take place at low bulk speeds. Values of $n_{\alpha} / n_{p}<0.005$ have been observed by Explorer 43 (Ogilvie and Burlaga, 1971), and such low values do not occur anywhere on the theoretical curves. For example on 29 March 1971, between 1730 and $1900 \mathrm{UT}$, the solar wind bulk speed was $330 \mathrm{~km} \mathrm{sec}$, the proton temperature $2.5 \times 10^{4} \mathrm{O}_{\mathrm{K}}$, and the proton density $\sim 4.5 \mathrm{~cm}^{-3}$. The relative abundance of helium was less than 0.005 for the entire period.

The $\left(\mathrm{n}_{\alpha} / \mathrm{n}_{\mathrm{p}}, \mathrm{T}_{\mathrm{p}}\right)$ relation, which shows some evidence of having the same form independent of solar activity, together with the $\left(U, T_{p}^{1 / 2}\right)$ relation, which is independent of solar activity, hold on the average over a wide range of 
conditions, and are constraints upon theories of the acceleration mechanism operating in the solar wind. Such a theory must show that both $n_{\alpha} / n_{p}$ and $T_{p}$ increase with increasing $U$. Weber (1971) has shown, using a hydrodynamical description of a hydrogen-helium plasma, that solar wind expansion requires the temperature of the helium to be about 3.5 times that of the hydrogen, as is normally observed. This model also predicts that a lower helium temperature is required for expansion when the helium to hydrogen mass flux ratio $n_{\alpha} U / n_{p} U$ is lower. Thus the value of $n_{\alpha} / n_{p}$ should be positively correlated with $T_{\alpha}$ or $T_{p}$, as observed here.

The study of these questions requires more, and more precise, data; that obtainable around solar minimum should be of particular interest. 


\section{References}

Burlaga, L。F. and Ogilvie, K. W., "Magnetic and thermal pressures in the solar wind" Solar Physics 15, 61, 1970b.

Burlaga, L. F. and Ogilvie, K. W., "Heating in the solar wind", Ap. J。 $159,659,1970$ a.

Geiss, J., Hirt, P., Leutwyler, $\mathrm{H}_{\circ}$, "On acceleration and motion of ions in corona and solar wind", Solar Physics, 12, 458, 1970.

Hirschberg, J., "Solar wind helium enrichments following major solar flares", Proc. Asilomar solar wind conference, Asilomar, Cal., 1971 to be published。

Hirschberg, J., Alksne, A., Colburn, D. S., Bame, S. J., and Hundhausen, A. J., "Observations of a solar flare induced interplanetary shock and helium enriched drivex gas", J。Geophys。Res。, 75, 1, 1970.

Hirschberg, Jo, Asbridge, J. Ro, and Robbins, D. E., "Velocity and flux dependence of the solar wind helium abundance", submitted to

J. Geophys。Res。, 1972。

Hundhausen, A. J。, "Composition and dynamics of solar wind plasma", Reviews of Geophysics and Space Science, $\underline{8}, 729,1970$.

Ness, N. F., Hundhausen, A. J。, Bame, S. J॰, "Observations of the interplanetary medium: Vela 3 and IMP 3, 1965-1967, J. Geophys. Res., 76, 6643, 1971 .

Ogilvie, K. Wo, Burlaga, L. F。, and Richardson, H., "Analysis of plasma measurements on IMP-F, NASA-GSFC X-612-67-543, 1967.

Ogilvie, K。 W., and Burlaga, L。F., "Solar wind composition observations", Trans. Am. Geophys. Union, 11, 887, 1971。

Ogilvie, K. W., and Zwally, J., "Hydrogen and helium velocities in the solar wind", submitted to Solar Physics, 1971.

Robbins, D。 E., Hundhausen, A。 J。, Bame, S。 J。, "Helium in the solar wind", J. Geophys. Res., 75, 1178, 1970。

Wang, J. R., "Dynamics of the eleven-year modulation of galactic cosmic rays', Ap. J., 160, 261, 1970.

Weber, E., "Hydrogen-helium expansion from the sun", Preprint, 1970. 


\section{Figure Captions}

Figure 1 The lowest line represents equation (1) derived by Hirschberg et. a1. (1972) from a least squares fit to the points shown, which are Vela $3 A$ and $3 B$ observations. The error bars indicate the standard error calculated assuming normal distribution. The center line represents equation (2), obtained by fitting to the circled points from Explorer 43 data. The uppermost 1 ine has the same slope as the others, but its intercept is obtained by fitting the triangle points, derived from Explorer 34 observations. The corresponding values of average sunspot number are indicated in each case. The point $M$ is plotted at the coordinates of the average values of bulk speed and $\mathrm{n}_{\alpha} / \mathrm{n}_{\mathrm{p}}$ for Mariner II.

Figure 2 The values of $B$ in equation (4), plotted at the corresponding values of average sunspot number.

Figure 3 The variation of $n_{\alpha} / n_{p}$ with solar wind flux Triangles - Exp. 34 data
Circled points - Exp. 43 data
Crosses :- Vela $3 \mathrm{~A}$ and $3 \mathrm{~B}$ data

Figure 4 This figure shows a plot of $n_{\alpha} / n_{p}$ vs. $T_{p}$. The dotted 1 ine represents the relation $\frac{\mathrm{n}_{\alpha}}{\mathrm{n}_{\mathrm{p}}} \times 10^{2}=0.28\left(\mathrm{~T}_{\mathrm{p}}^{1 / 2}\right)+0.08 \mathrm{~S}-2.37, \mathrm{~T}_{\mathrm{p}}$ in kilodegrees, evaluated for $S=50$. The circled points are Explorer 43 data, corrected from $S=65$ to $S=50$, and the triangles are Explorer 34 data, corrected from $S=93$ to $S=50$. The crosses are Vela data. 


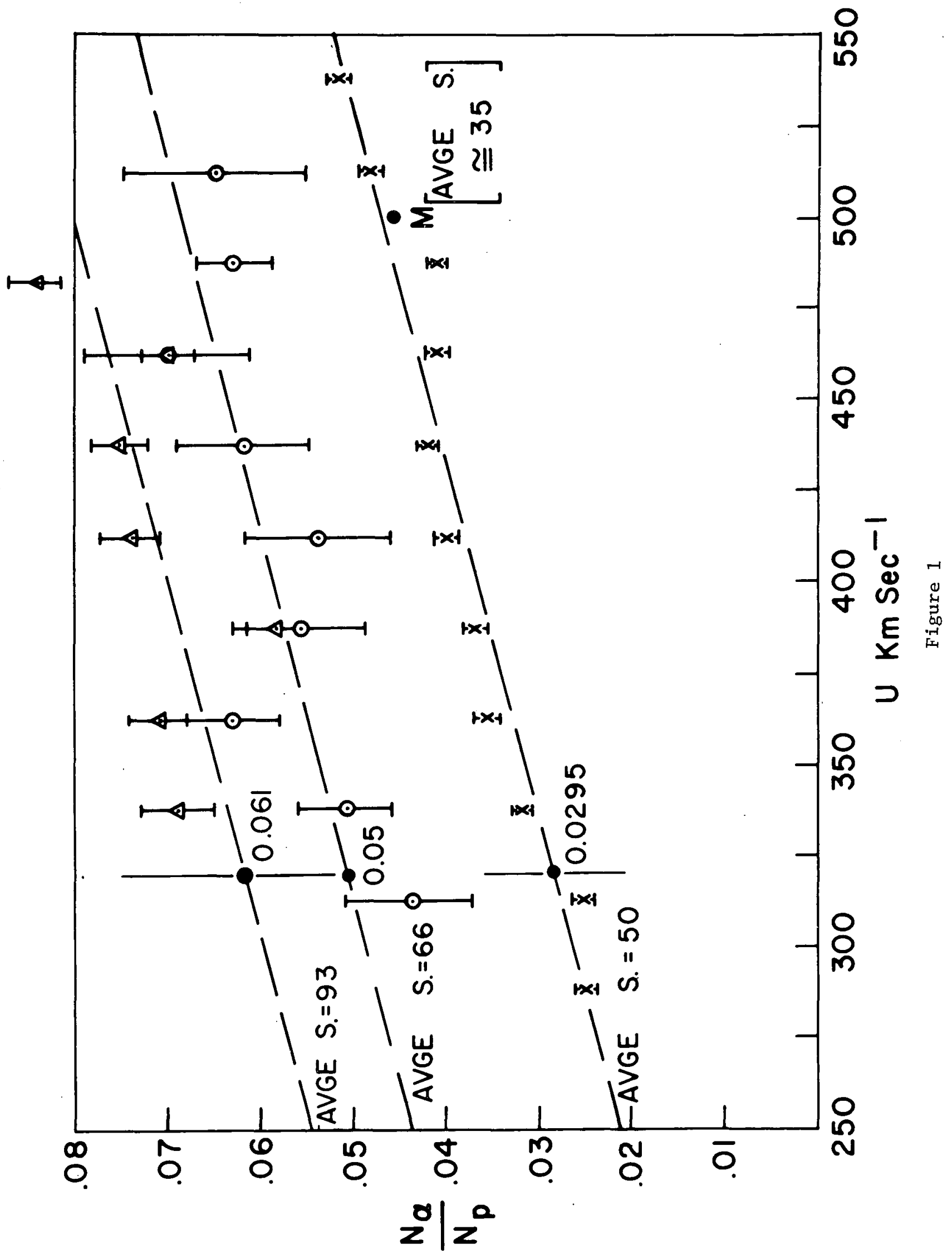




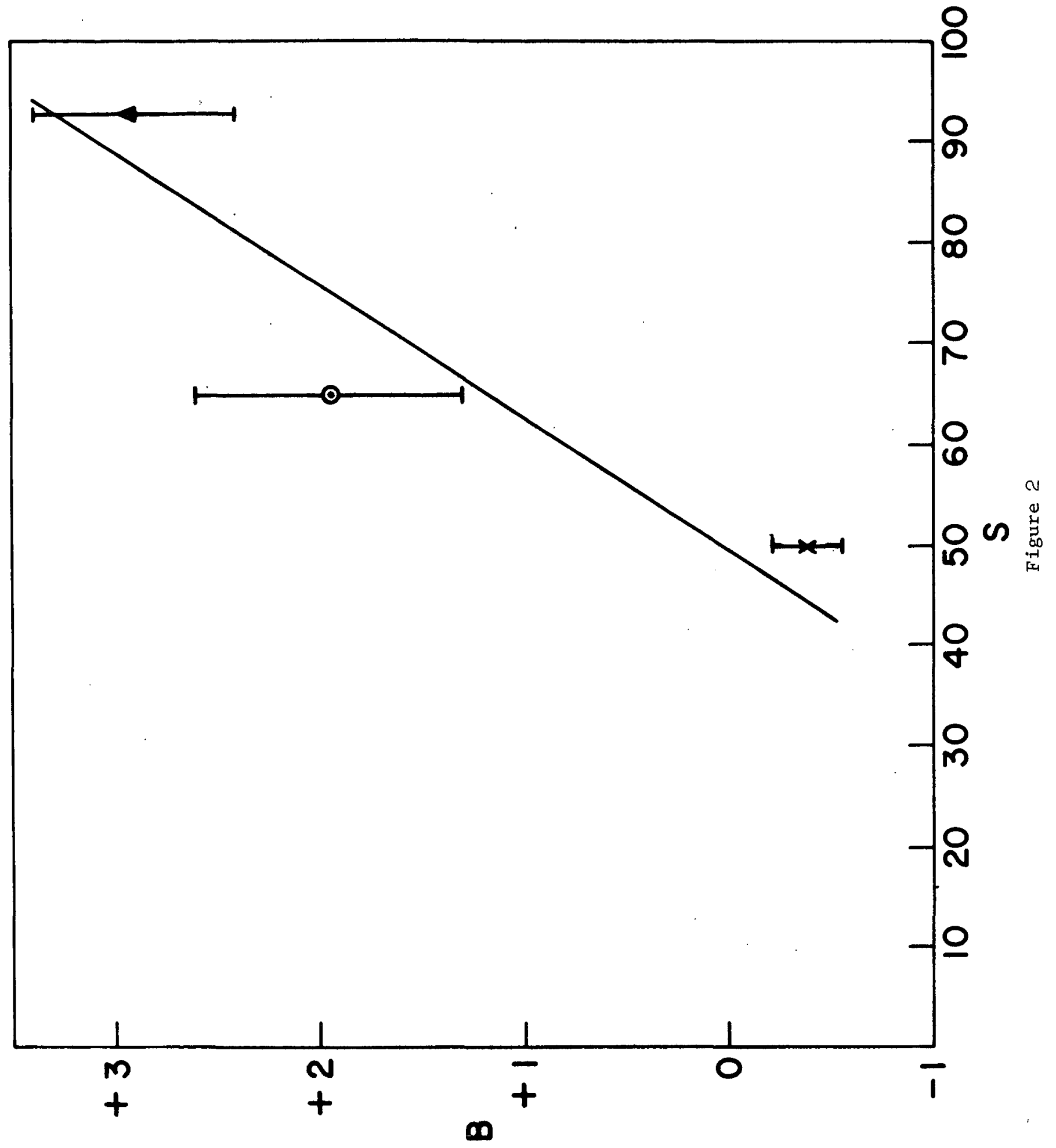




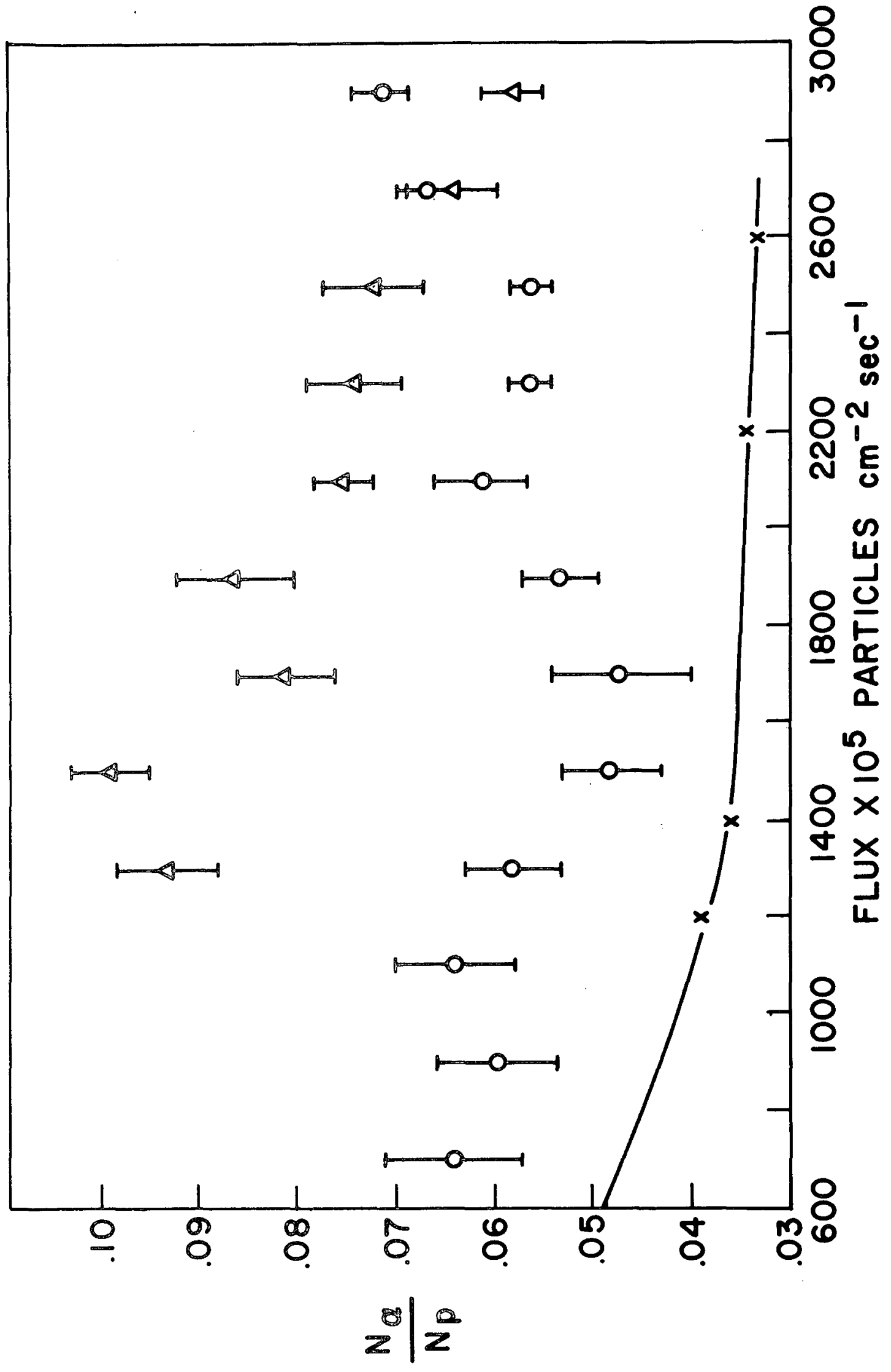




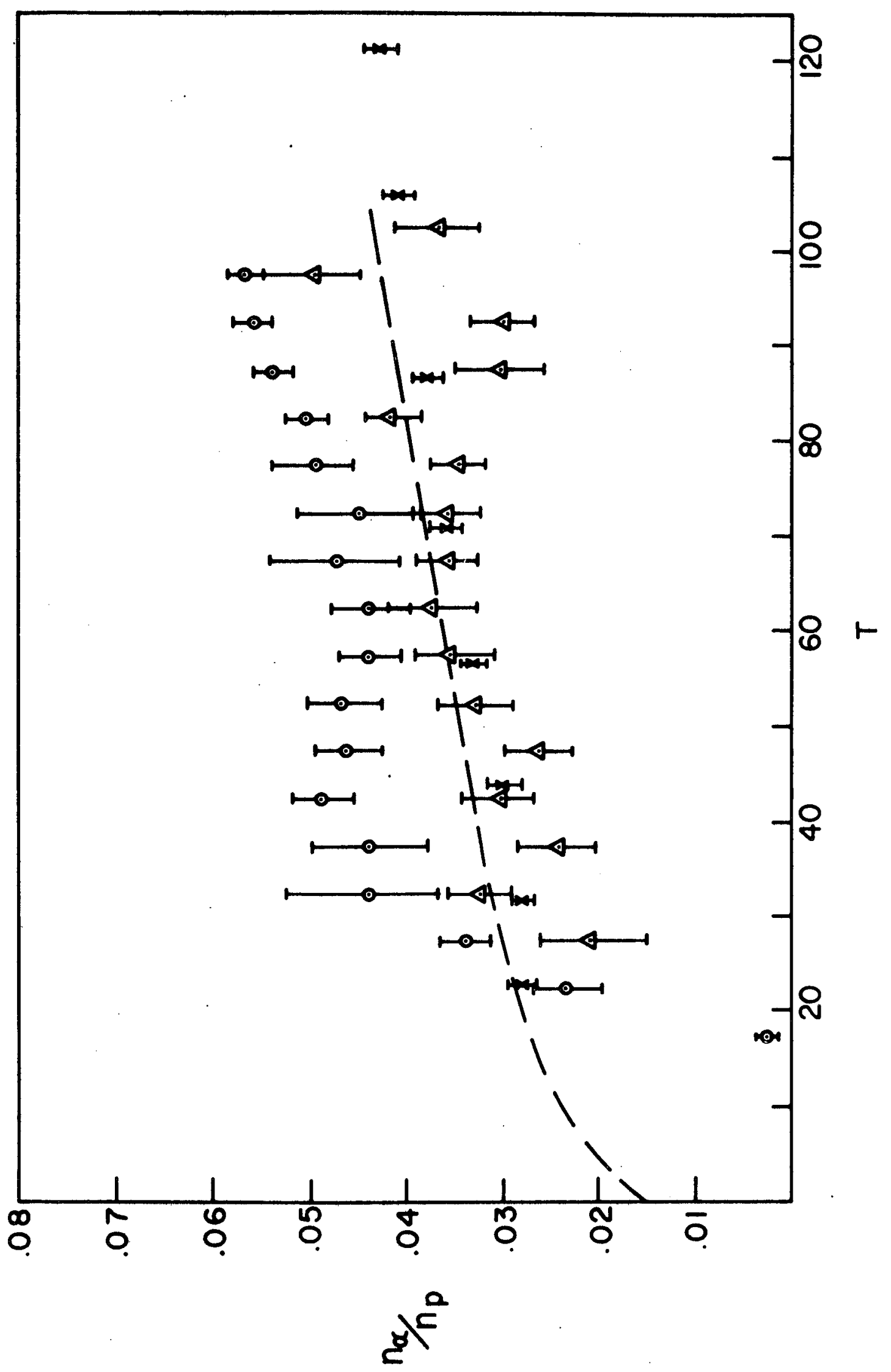

